Vasile Ene, Quellenstraße 18, 63571 Gelnhausen, Germany. e-mail:

gabrielaene@hotmail.com

\title{
AN IMPROVEMENT OF A RECENT RESULT OF THOMSON
}

\begin{abstract}
In [5], Brian S. Thomson proved the following result: Let $f$ be $A C^{*} G$ on an interval $[a, b]$. Then the total variation measure $\mu=\mu_{f}$ associated with $f$ has the following properties: a) $\mu$ is a $\sigma$-finite Borel measure on $[a, b]$; b) $\mu$ is absolutely continuous with respect to Lebesgue measure; c) There is a sequence of closed sets $F_{n}$ whose union is all of $[a, b]$ such that $\mu\left(F_{n}\right)<\infty$ for each $n$; d) $\mu(B)=\mu_{f}(B)=\int_{B}\left|f^{\prime}(x)\right| d x$ for every Borel set $B \subset[a, b]$. Conversely, if a measure $\mu$ satisfies conditions a) $-c)$ then there exists an $A C^{*} G$ function $f$ for which the representation d) is valid. In this paper we improve Thomson's theorem as follows: in the first part we ask $f$ to be only $V B^{*} G \cap(N)$ on a Lebesgue measurable subset $P$ of $[a, b]$ and continuous at each point of $P$; the converse is also true even for $\mu$ defined on the Lebesgue measurable subsets of $P$ (see Theorem 2 and the two examples in Remark 1).
\end{abstract}

In [5] Brian S. Thomson proved a theorem that can be written in the following form:

\section{Theorem A.}

I. If $F:[a, b] \rightarrow \mathbb{R}$ is $A C^{*} G$ on $[a, b]$ then $\mu_{F}^{*}: \mathcal{P}([a, b]) \rightarrow[0,+\infty]$ has the following properties:

1) $\mu_{F}^{*} \ll m$;

2) there is a sequence of closed sets $\left\{P_{n}\right\}$ such that $\cup_{n=1}^{\infty} P_{n}=[a, b]$ and $\mu_{F}^{*}\left(P_{n}\right)<+\infty$ for each $n$.

3) $\left(\mu_{F}^{*}\right)_{\mid \mathcal{B o r}([a, b])}$ is a measure (see [4, p. 40]);

4) $\mu_{F}^{*}(B)=(\mathcal{L}) \int_{B}\left|F^{\prime}(t)\right| d t$ whenever $B$ is a Borel subset of $[a, b]$.

Key Words: variational measure, Borel sets, Lebesgue sets, $V B^{*} G, A C^{*} G$, Lusin's condition $(N)$

Mathematical Reviews subject classification: 26A45, 26A39, 28A12

Received by the editors October 22, 1998

*The author died on November 11, 1998; see Real Anal. Exch. 241 (1998/99), 3. 
II. Conversely, let $\mu: \mathcal{B}$ or $([a, b]) \rightarrow[0,+\infty]$ be a measure such that:

$\left.1^{\prime}\right) \mu \ll m$;

$\left.2^{\prime}\right)$ there is a sequence of closed sets $\left\{P_{n}\right\}$ such that $\cup_{n=1}^{\infty} P_{n}=[a, b]$ and $\mu\left(P_{n}\right)<+\infty$ for each $n$;

Then there exists a continuous function $F:[a, b] \rightarrow \mathbb{R}, F \in A C^{*} G$ on $[a, b]$, such that $\left(\mu_{F}^{*}\right)_{\mid \mathcal{B} \text { or }([a, b])}=\mu$.

In this paper we shall improve Theorem A as it will be shown in Theorem 2 (see also the two examples given in Remark 1).

We denote by $m$ the Lebesgue measure in $\mathbb{R}$. By $\mathcal{O}(f ; X)$ we shall mean the oscillation of the function $f$ on the set $X$, and by $f_{\mid X}$ the restriction of the function $f$ on the set $X$. The conditions $A C, A C G, A C^{*} A C^{*} G, V B^{*}$, $V B^{*} G$ and Lusin's condition $(N)$ are defined as in [3].

Definition 1. Let $P \subset \mathbb{R}, \mathcal{A} \subseteq \mathcal{P}(P)=\{E: E \subset P\}$ and $\alpha: \mathcal{A} \rightarrow[0,+\infty]$.

- We say that $\alpha$ is absolutely continuous with respect to $m$ and write $\alpha \ll m$ if $\alpha(Z)=0$ whenever $Z \in \mathcal{A}$ and $m(Z)=0$.

- For $P$ a Lebesgue measurable subset of $\mathbb{R}$, we put $\mathcal{L} e b(P)=\{E \subset P: E$ is Lebesgue measurable\}.

- For $P$ a Borel measurable subset of $\mathbb{R}$, we put $\operatorname{Bor}(P)=\{E \subset P: E$ is Borel measurable\}.

Definition 2. For $x, y \in \mathbb{R}, x \neq y$, let $\langle x, y\rangle$ denote the closed interval with the endpoints $x$ and $y$. Let $E \subset \mathbb{R}, \delta: E \rightarrow(0,+\infty)$,

$$
\beta^{*}(E ; \delta)=\{(\langle x, y\rangle, x): x \in E, y \in(x-\delta(x), x+\delta(x))\} .
$$

The finite set $\pi=\left\{\left(\left\langle x_{i}, y_{i}\right\rangle, x_{i}\right)\right\}_{i=1}^{n} \subset \beta^{*}(E ; \delta)$ is said to be a partition if $\left\{\left\langle x_{i}, y_{i}\right\rangle\right\}_{i=1}^{n}$ is a set of nonoverlapping closed intervals. Let $f: \mathbb{R} \rightarrow \mathbb{R}$,

$$
V_{\delta}^{*}(f ; E)=\sup \left\{\sum_{(\langle x, y\rangle, x) \in \pi}|f(y)-f(x)|: \pi \subset \beta^{*}(E ; \delta) \text { is a partition }\right\},
$$

and

$$
\mu_{f}^{*}(E)=\inf _{\delta} V_{\delta}^{*}(f ; E) .
$$

Note that this $\mu_{f}^{*}$ is the same as that of Thomson [5, p. 186], and it is also identical with Thomson's $\mathcal{S}_{o}-\mu_{F}$ of [4]. 
Lemma 1. Let $F:[a, b] \rightarrow \mathbb{R}, E \subset P \subset[a, b], F \in V B^{*}$ on $E, F$ continuous at each point of $P$. Then $\mu_{F}^{*}(\bar{E} \cap P) \neq+\infty$.

Proof. By Theorem 7.1 of [3, p. 229], $F$ is $V B^{*}$ on $\bar{E} \cap P$. Let $X=\bar{E} \cap P$ and $Y=\{x \in X: x$ is isolated at least at one side in $X\}$. Since $Y$ is at most countable [3, p. 260], and $F$ is continuous at each point of $P$, it follows that $\mu_{F}^{*}(Y)=0$. Thomson shows in $\left[4\right.$, p. 34] that $\mu_{F}^{*}(X \backslash Y) \leq 2 V^{*}(F ; X)$. Hence $\mu_{F}^{*}(X) \leq 2 V^{*}(F ; X) \neq+\infty$.

Theorem 1. Let $F:[a, b] \rightarrow \mathbb{R}$, and let $P$ be a Lebesgue measurable subset of $[a, b]$. Let $\mu_{F}^{*}: \mathcal{P}(P) \rightarrow[0,+\infty]$. The following assertions are equivalent:

(i) $\mu_{F}^{*} \ll m$;

(ii) $F$ is $V B^{*} G \cap(N)$ on $P$ and $F$ is continuous at each point of $P$;

(iii) $F$ is continuous at each point of $P$, derivable a.e. on $P$, and

$$
\mu_{F}^{*}(E)=(\mathcal{L}) \int_{E}\left|F^{\prime}(t)\right| d t
$$

whenever $E$ is a Lebesgue measurable subset of $P$;

Moreover, each of the three equivalences implies that there exists a sequence of sets $P_{n}$ such that $\cup_{n} P_{n}=P$ and $\mu_{F}^{*}\left(\bar{P}_{n} \cap P\right) \neq+\infty$.

Proof. The three equivalences follow from [2, Theorem 13, (ii), (iii), (vii)] (because $\mathcal{S}_{o}-\mu_{F}=\mu_{F}^{*}$ ). The second part follows by Lemma 1 and (ii).

Lemma 2. Let $f:[a, b] \rightarrow[0,+\infty)$ a Lebesgue integrable function, $P$ a closed subset of $[a, b],\left\{\left(a_{i}, b_{i}\right)\right\}_{i}$ the intervals continuous to $P \cup\{a, b\}$, and let $\left\{\alpha_{i}\right\}_{i}$ be a sequence of positive numbers. Then there is a function $G:[a, b] \rightarrow \mathbb{R}$ such that:

a) $G(t)=0$ for $t \in P \cup\{a, b\}$;

b) $G \in A C$ on $[a, b]$;

c) $\left|G^{\prime}(t)\right|=f(t)$ a.e. on $\cup_{i=1}^{\infty}\left(a_{i}, b_{i}\right)$;

d) $G(t) \in\left[0, \alpha_{i}\right)$ for $t \in\left[a_{i}, b_{i}\right], i=1,2, \ldots$;

e) $G^{\prime}(t)=0$ a.e. on $P$. 
Proof. We shall use a technique of Thomson [5, p. 190]. For each $i$, let $n_{i}$ be a positive integer, and let

$$
a_{i}=a_{i, 0}<a_{i, 1}<a_{i, 2}<\ldots<a_{i, 2 n_{i}-1}<a_{i, 2 n_{i}}=b_{i}
$$

be such that

$$
\int_{a_{i, k}}^{a_{i, k+1}} f(t) d t=\frac{1}{2 n_{i}} \int_{a_{i}}^{b_{i}} f(t) d t<\alpha_{i} .
$$

Let $g:[a, b] \rightarrow \mathbb{R}$

$$
g(t)= \begin{cases}0 & \text { if } t \in P \cup\{a, b\} \\ f(t) & \text { if } t \in\left[a_{i, 2 k}, a_{i, 2 k+1}\right], k=\overline{0, n_{i}-1}, i=\overline{1, \infty} \\ -f(t) & \text { if } t \in\left(a_{i, 2 k-1}, a_{i, 2 k}\right), k=\overline{1, n_{i}}, i=\overline{1, \infty} .\end{cases}
$$

Then $G:[a, b] \rightarrow \mathbb{R}, G(x)=\int_{a}^{x} g(t) d t$ satisfies our lemma.

Lemma 3. Let $f, f_{n}:[a, b] \rightarrow \mathbb{R}$ be such that the series $\sum_{n=1}^{\infty} f_{n}(x)=f(x)$ for $x \in[a, b]$. Then

$$
\mathcal{O}(f ;[a, b]) \leq \sum_{n=1}^{\infty} \mathcal{O}\left(f_{n} ;[a, b]\right)
$$

Proof. Let $x, y \in[a, b]$. Then

$$
|f(y)-f(x)|=\left|\sum_{n=1}^{\infty}\left(f_{n}(y)-f_{n}(x)\right)\right| \leq \sum_{n=1}^{\infty}\left|f_{n}(y)-f_{n}(x)\right| \leq \sum_{n=1}^{\infty} \mathcal{O}\left(f_{n} ;[a, b]\right) .
$$

Thus we have (1).

Theorem 2. Let $P$ be a Lebesgue measurable subset of $[a, b]$.

I. If $F:[a, b] \rightarrow \mathbb{R}$ is $V B^{*} G \cap(N)$ (particularly $F \in A C^{*} G$ ) on $P$ and $F$ is continuous at each point of $P$, then $\mu_{F}^{*}: \mathcal{P}(P) \rightarrow[0,+\infty]$ has the following properties:

1) $\mu_{F}^{*} \ll m$

2) there is a a sequence of sets $P_{n}$ such that $\cup_{n} P_{n}=P$ and for each $n$, $\mu_{F}^{*}\left(\bar{P}_{n} \cap P\right) \neq+\infty$;

3) $\left(\mu_{F}^{*}\right)_{\mid \mathcal{L} e b(P)}$ is a measure; 
4) $\mu_{F}^{*}(B)=(\mathcal{L}) \int_{B}\left|F^{\prime}(t)\right| d t$ whenever $B \subset \mathcal{L} e b(P)$.

II. Conversely, let $\mu: \mathcal{L} e b(P) \rightarrow[0,+\infty]$ be a measure such that:

$\left.1^{\prime}\right) \mu \ll m ;$

$\left.2^{\prime}\right)$ there is a sequence of sets $P_{n}$ such that $\cup_{n} P_{n}=P$ and for each $n$, $\mu\left(\bar{P}_{n} \cap P\right) \neq+\infty$.

Then there exists a continuous function $F:[a, b] \rightarrow \mathbb{R}, F \in A C^{*} G$ on $P$, such that $\left(\mu_{F}^{*}\right)_{\mid \mathcal{L e b}(P)}=\mu$.

Proof. I. 1) follows by Theorem 1, (i), (ii).

2) follows by the last part of Theorem 1.

3) Let $\left\{E_{n}\right\}_{n} \subset \mathcal{L} e b(P)$ be a sequence of pairwise disjoint sets. Then each $E_{n}=A_{n} \cup B_{n}$, where $A_{n}$ is a Borel set and $m\left(B_{n}\right)=0$. By 1$), \mu_{F}^{*}\left(B_{n}\right)=0$. Since $\mu_{F}^{*}$ is a metric outer measure it follows that $\mu_{F}^{*}$ restricted to the Borel subsets of $[a, b]$ is a measure. Thus we obtain:

$$
\begin{aligned}
\mu_{F}^{*}\left(\cup_{n} E_{n}\right) & \leq \sum_{n}^{\infty} \mu_{F}^{*}\left(E_{n}\right) \leq \sum_{n}^{\infty}\left(\mu_{F}^{*}\left(A_{n}\right)+\mu_{F}^{*}\left(B_{n}\right)\right) \\
& =\sum_{n}^{\infty} \mu_{F}^{*}\left(A_{n}\right)=\mu_{F}^{*}\left(\cup_{n} A_{n}\right) \leq \mu_{F}^{*}\left(\cup_{n} E_{n}\right) .
\end{aligned}
$$

Thus $\left(\mu_{F}^{*}\right)_{\mid \mathcal{L} e b(P)}$ is a measure.

4) See Theorem 1, (ii), (iii).

II. Let $Q_{0}=\emptyset$ and $Q_{n}=\cup_{i=1}^{n} \bar{P}_{i} \cup\{a, b\}, n=1,2, \ldots$ For $n \geq 1$, let $\left\{\left(a_{n j}, b_{n j}\right)\right\}$ be the intervals contiguous to $Q_{n}$. Clearly $\mu\left(Q_{n} \cap P\right) \neq+\infty$. We shall use Thomson's technique of [5, p. 189-190]. Since $\mu$ is absolutely continuous on $P \cap\left(Q_{n} \backslash Q_{n-1}\right)$ and $\mu\left(P \cap\left(Q_{n} \backslash Q_{n-1}\right)\right) \neq+\infty$, by the Radon-Nicodym Theorem, there exists a Lebesgue integrable function $g_{n}$ : $P \cap\left(Q_{n} \backslash Q_{n-1}\right) \rightarrow[0,+\infty)$ such that

$$
\mu(B)=(\mathcal{L}) \int_{B} g_{n}(t) d t
$$

whenever $B$ is a Lebesgue measurable subset of $P \cap\left(Q_{n} \backslash Q_{n-1}\right)$. We may consider $g_{n}:[a, b] \rightarrow \mathbb{R}$, if we put $g_{n}(x)=0$ for $x \in[a, b] \backslash\left(P \cap\left(Q_{n} \backslash Q_{n-1}\right)\right)$. Let

$$
F_{1}(x)=(\mathcal{L}) \int_{a}^{x} g_{1}(t) d t
$$


Then $F_{1} \in A C$ on $[a, b]$ and $F_{1}^{\prime}=g_{1}$ a.e. on $[a, b]$. Clearly $F_{1}$ is constant on each $\left(a_{1 j}, b_{1 j}\right)$. Let $\left\{\alpha_{n j}\right\}_{j}$ be a sequence of positive numbers such that

$$
\sum_{j=1}^{\infty} \alpha_{n j}<\frac{1}{2^{n}}
$$

By Lemma 2, there exists $F_{n+1}:[a, b] \rightarrow\left[0, \frac{1}{2^{n}}\right)$ such that

a) $F_{n+1}(t)=0$ for $t \in Q_{n}$;

b) $F_{n+1} \in A C$ on $[a, b]$;

c) $\left|F_{n+1}^{\prime}(t)\right|=g_{n+1}(t)$ a.e. on each $\left(a_{n j}, b_{n j}\right)$;

d) $F_{n+1}(t) \in\left[0, \alpha_{n j}\right)$ on $\left[a_{n j}, b_{n j}\right]$;

e) $F_{n+1}^{\prime}(t)=0$ a.e. on $Q_{n}$.

Let $F:[a, b] \rightarrow \mathbb{R}, F(x)=\sum_{n=1}^{\infty} F_{n}(x)$. Then $F$ is continuous on $[a, b]$ (see d), b) and (2)). Let $R_{n}(x)=\sum_{k=1}^{\infty} F_{n+k}(x)$. Since each

$$
\left(a_{n j}, b_{n j}\right) \subset[a, b] \backslash Q_{n} \subset[a, b] \backslash\left(P \cap\left(Q_{n} \backslash Q_{n-1}\right)\right),
$$

it follows that $g_{n}(t)=0$ on $\left(a_{n j}, b_{n j}\right)$. By c) we have that $F_{n}^{\prime}(t)=0$, so $F_{n}$ is constant on each $\left(a_{n j}, b_{n j}\right)$. Thus

$$
F_{1}(x)+\ldots+F_{n}(x)=\text { constant on each }\left(a_{n j}, b_{n j}\right)
$$

(because $Q_{1} \subset Q_{2} \subset \ldots$ ). Since $F_{n+k}(t)=0$ on $Q_{n+k-1}$ for $k=\overline{1, \infty}$ (see a)), and $Q_{n} \subset Q_{n+1} \subset Q_{n+2} \subset \ldots$ it follows that $R_{n}(t)=0$ on $Q_{n}$. Thus $F(x)=F_{1}(x)+\ldots+F_{n}(x)$ for $x \in Q_{n}$. Hence $F$ and $R_{n}$ are $A C$ on $Q_{n}$. By Lemma 3 and (3), we have

$$
\begin{aligned}
\sum_{j} \mathcal{O}\left(F ;\left[a_{n j}, b_{n j}\right]\right) & =\sum_{j} \mathcal{O}\left(R_{n} ;\left[a_{n j}, b_{n j}\right]\right) \\
& \leq \sum_{j}\left(\mathcal{O}\left(F_{n+1} ;\left[a_{n j}, b_{n j}\right]\right)+\mathcal{O}\left(F_{n+2} ;\left[a_{n j}, b_{n j}\right]\right)+\ldots\right) \\
& =\sum_{j} \mathcal{O}\left(F_{n+1} ;\left[a_{n j}, b_{n j}\right]\right)+\sum_{j} \mathcal{O}\left(F_{n+2} ;\left[a_{n j}, b_{n j}\right]\right)+\ldots \\
& <\frac{1}{2^{n}}+\sum_{j} \mathcal{O}\left(F_{n+2} ;\left[a_{n+1, j}, b_{n+1, j}\right]\right)+\ldots \\
& <\frac{1}{2^{n}}+\frac{1}{2^{n+1}}+\ldots=\frac{1}{2^{n-1}} .
\end{aligned}
$$


(see a), d) and (2)). By [3, p. 232], $F$ and $R_{n}$ are $A C^{*}$ on $Q_{n}$. Clearly $R_{n}^{\prime}(x)=0$ a.e. on $Q_{n}$ and $F$ is $A C^{*} G\left(\subset V B^{*} G \cap(N)\right)$ on $P$. It follows that

$$
F^{\prime}(x)=F_{1}^{\prime}(x)+\ldots+F_{n}^{\prime}(x)+R_{n}^{\prime}(x)=F_{1}^{\prime}(x)+\ldots+F_{n}^{\prime}(x) \text { a.e. on } Q_{n} .
$$

Thus $F^{\prime}(x)=F_{1}^{\prime}(x)=g_{1}(x)$ on $Q_{1}$ and

$$
\left|F^{\prime}(x)\right|=\left|F_{1}^{\prime}(x)+F_{2}^{\prime}(x)\right|=\left|F_{2}^{\prime}(x)\right|=g_{2}(x) \text { on } Q_{2} \backslash Q_{1}
$$

(because $F_{1}$ is constant on each $\left.\left(a_{1 j}, b_{1 j}\right)\right)$. Continuing, it follows that

$$
\left|F^{\prime}(x)\right|=\left|F_{1}^{\prime}(x)+\ldots+F_{n-1}^{\prime}(x)+F_{n}^{\prime}(x)\right|=\left|F_{n}^{\prime}(x)\right|=g_{n}(x) \text { on } Q_{n} \backslash Q_{n-1}
$$

(because $F_{1}, \ldots, F_{n-1}$ are constant on each $\left.\left(a_{n-1, j}, b_{n-1, j}\right)\right)$. By 3$)$, for any Lebesgue measurable subset $B$ of $P$, we have

$$
\begin{gathered}
\mu_{F}^{*}(B)=\sum_{n=1}^{\infty} \mu_{F}^{*}\left(B \cap\left(Q_{n} \backslash Q_{n-1}\right)\right)=\sum_{n=1}^{\infty}(\mathcal{L}) \int_{B \cap\left(Q_{n} \backslash Q_{n-1}\right)}\left|F^{\prime}(t)\right| d t= \\
=\sum_{n=1}^{\infty}(\mathcal{L}) \int_{B \cap\left(Q_{n} \backslash Q_{n-1}\right)} g_{n}(t) d t=\sum_{n=0}^{\infty} \mu\left(B \cap\left(Q_{n} \backslash Q_{n-1}\right)\right)=\mu(B) .
\end{gathered}
$$

Thus $\mu_{F}^{*}(B)=\mu(B)$.

\section{Remark 1.}

- Theorem 2 contains Theorem A of Thomson.

- We recall the following example of [2]:

Let $C$ be the Cantor ternary set and $\varphi:[0,1] \rightarrow[0,1]$ the Cantor ternary function (see for example [1], pp. 213-214). Then $C$ contains a $G_{\delta}$-set $B$ such that $m^{*}(\varphi(B))=0$, hence $\varphi \in V B^{*} G \cap(N)$ on $B$. But $\varphi \notin A C G$ on $B$, so $\varphi \notin A C^{*} G$ on $B$.

From this example it follows that $A C^{*} G \subsetneq V B^{*} G \cap(N)$, so in Theorem 2, I., the particular case with $A C^{*} G$ is genuine. Moreover $\mu_{\varphi}^{*}=\mu_{f}^{*}=0$ on $\mathcal{L} e b(B)$ whenever $f:[0,1] \rightarrow \mathbb{R}$ is $V B^{*} G \cap(N)$ on $B$ and continuous at each point of $B$ (see Theorem 1).

- We consider the following example: 
Let $C$ be the Cantor ternary set. Let $\left\{\left(a_{n i}, b_{n i}\right)\right\}, n=1,2, \ldots, i=$ $1,2, \ldots, 2^{n-1}$, be the intervals contiguous to $C$ of length $\frac{1}{3^{n}}$, and let $c_{n i}=\frac{a_{n i}+b_{n i}}{2}$. Let $F:[0,1] \rightarrow[0,1]$,

$$
F(x)= \begin{cases}0 & \text { if } x \in C \\ \frac{1}{n} & \text { if } x=c_{n i} \\ \text { linear } & \text { on each }\left[a_{n i}, c_{n i}\right] \text { and }\left[c_{n i}, b_{n i}\right]\end{cases}
$$

Let $P=\cup_{n=1}^{\infty} \cup_{i=1}^{2^{n-1}}\left(a_{n i}, b_{n i}\right)$. Clearly $F$ is continuous on $[a, b], F$ is $A C^{*} G$ on $P$, but $F$ is not $A C^{*} G$ on $[0,1]$.

This example shows that the particular case of Theorem 2, I., also strictly contains Thomson's Theorem A, I. because our theorem holds for the function $F$, but Thomson's theorem doesn't.

\section{References}

[1] V. Ene, Real functions - current topics, Lect. Notes in Math., vol. 1603, Springer-Verlag, 1995.

[2] V. Ene, Thomson's variational measure, Real Analysis Exchange 24(199899), no. 2, 523-566.

[3] S. Saks, Theory of the integral, 2nd. rev. ed., vol. PWN, Monografie Matematyczne, Warsaw, 1937.

[4] B. S. Thomson, Real functions, Lect. Notes in Math., vol. 1170, SpringerVerlag, 1985.

[5] B. S. Thomson, $\sigma$-finite Borel measures on the real line, Real Analysis Exchange 23(1997-98), no. 1, 185-192. 